\title{
Substantial reduction in caries from regular fluoride varnish application
}

\author{
Abstracted from \\ Marinho VCC, Worthington HV, Walsh T, Clarkson JE. \\ Fluoride varnishes for preventing dental caries in children and adolescents. \\ Cochrane Database Syst Rev 2013; Issue 7. Art. No.: CD002279. DOI: 10.1002/14651858.CD002279.pub2. \\ Address for correspondence: Luisa Fernandez Mauleffinch, Review Group Co-ordinator, \\ Cochrane Oral Health Group, MANDEC, School of Dentistry, University of Manchester, \\ Higher Cambridge Street, Manchester, M15 6FH, UK. E-mail: luisa.fernandez@manchester.ac.uk
}

\section{Question: How effective is fluoride varnish in preventing dental caries?}

Data sources The Cochrane Oral Health Group's Trials Register, the Cochrane Central Register of Controlled Trials (CENTRAL), Medline, Embase, CINAHL, LILACS and BBO, ProQuest Dissertations and Theses, Web of Science Conference Proceedings and ClinicalTrials. gov databases were searched. Reference lists of identified articles were also scanned for relevant papers. Study authors were contacted for additional information.

Study selection Randomised or quasi-randomised controlled trials using or indicating blind outcome assessment that compared fluoride varnish to placebo or no treatment for at least one year were included. Risk of bias assessment was undertaken.

Data extraction and synthesis Study assessment and data extraction was carried out independently by at least two reviewers. The primary measure of effect was the prevented fraction, that is the difference in mean caries increments between the treatment and control groups expressed as a percentage of the mean increment in the control group. The caries increments nearest to three years were used from each included study. Random-effects meta-analyses were performed where data could be pooled. Potential sources of heterogeneity were examined in random-effects meta-regression analyses. Adverse effects information was collected from the included trials.

Results Twenty-two trials (12,455 participants) were included. Thirteen trials were included in the permanent tooth surfaces meta-analysis, the pooled D(M)FS prevented fraction estimate comparing fluoride varnish with placebo or no treatment was $43 \%$ (95\% confidence interval $(\mathrm{Cl}) 30 \%$ to $57 \% ; \mathrm{P}<0.0001$ ). There was substantial heterogeneity, confirmed statistically $\left(\mathrm{P}<0.0001 ; \mathrm{I}^{2}=\right.$ $75 \%)$, however this body of evidence was assessed as of moderate quality. Ten trials contributed to the meta-analysis of primary teeth, the pooled $\mathrm{d}(\mathrm{e} / \mathrm{m}) \mathrm{fs}$ prevented fraction estimate was $37 \%(95 \% \mathrm{Cl} 24 \%$ to $51 \% ; \mathrm{P}<0.0001)$, there was some heterogeneity $\left(\mathrm{P}=0.009 ; \mathrm{I}^{2}=\right.$ $59 \%$ ) this evidence was assessed as of moderate quality. No significant associations in either dentitions were found with baseline caries

This paper is based on a Cochrane Review published in the Cochrane Library 2013, issue 7 (see www.thecochranelibrary.com for information). Cochrane Reviews are regularly updated as new evidence emerges and in response to feedback, and the Cochrane Library should be consulted for the most recent version of the review. severity, background exposure to fluorides, application features such as prior prophylaxis, concentration of fluoride or frequency of application. There was little information concerning possible adverse effects or acceptability of treatment.

Conclusions The conclusions of this updated review remain the same as when it was first published. The review suggests a substantial caries inhibiting effect of fluoride varnish in both permanent and primary teeth; however the quality of the evidence was assessed as moderate, as it included mainly high risk of bias studies, with considerable heterogeneity.

\section{Commentary}

Fluoride varnishes have been available since the 1960s and were developed to prolong the contact time between fluoride and enamel. There are several different formulations on the market with the main commercially available varnishes containing $5 \%$ sodium fluoride 22,600 parts per million fluoride ions (ppm F).

While caries prevalence has fallen in many countries it still affects significant proportions of children and adolescents. Increasing the availability of fluoride is a key element in the prevention of caries, and the use of topical fluoride agents, toothpastes (dentifrices), mouthrinses, gels and varnishes for prevention is important. The earlier version of this review highlighted its substantial caries-inhibiting effect in both the primary and permanent dentition. ${ }^{1}$ This has had an important impact, with the use of fluoride varnish being incorporated into a number of evidence-based guidelines. ${ }^{3-5}$ This has led to its increased use on an individual and community level to reduce caries.

Since the 2002 review there have been three subsequent reviews of fluoride varnish by other authors. ${ }^{6-8}$ While these have supported the findings of the original Cochrane they have included study designs other than randomised controlled trials (RCTs). These reviews also only include a small number of trials, the maximum being four. ${ }^{7}$ This update follows the usual robust Cochrane methodology, with an extensive search of relevant databases together with some additional hand searching of relevant journals. The search was restricted to random or quasi-randomised trials with robust outcome assessment.

Twenty-two trials involving 12,455 participants were included, adding 13 additional trials to the earlier version of the review. The main finding was that there was on average a $43 \%$ reduction in decayed, missing and filled tooth surfaces for permanent teeth, with a $95 \%$ confidence interval (CI) of $30-57 \%$. For primary teeth 
surfaces the figure was 37\% ( 95\% CI 24-51\% ). Similar reductions were evident for permanent and primary teeth at 44\% (95\% CI -11$76 \%$ ) and $65 \%$ (95\%CI- 48-82\%) respectively. These are substantial reductions in dental disease and should serve to reinforce the role of fluoride varnish as a key preventive tool for both individual and community based prevention programmes.

In addition to the main finding the authors also looked at whether the effectiveness of the varnish was affected by the initial level of caries severity, background exposure to fluoride, concentration of fluoride, frequency of application and whether prophylaxis was undertaken prior to the application of the varnish. The regression analysis conducted suggests that these factors did not have an effect although they do point out that only small numbers of trials were included in this analysis so it should be interpreted cautiously. In terms of the application frequency 17 of the studies tested two applications per year with only three using four applications per year. Two applications per year is what is recommended in most guidelines, with some recommending four for high caries risk individuals.

There was little information available on possible adverse effects or acceptability of treatment. As some of the varnishes have colophony (or rosin - a sticky substance obtained from conifers) as a component which is a known allergen, ${ }^{9}$ it is important that new studies should be better at the reporting of adverse effects, although to date, only one case in an adult appears to have been reported in the literature. ${ }^{10}$

\section{Practice point}

- The use of fluoride varnish two to four times per year is associated with a substantial reduction in caries.

\section{Derek Richards}

Centre for Evidence-based Dentistry, Oxford, UK

1. Marinho VCC, Higgins JPT, Logan S, Sheiham A. Fluoride varnishes for preventing dental caries in children and adolescents. Cochrane Database Syst Rev 2002; Issue 1. Art. No.: CD002279. DOI: 10.1002/14651858.CD002279.

2. SIGN 47. Preventing Dental Caries in Children at High Caries Risk. Targeted Prevention of Dental Caries in the Permanent Teeth of 6-16 Year Olds Presenting for Dental Care. Scottish Intercollegiate Guidelines Network (2000) (www.sign.ac.uk/ guidelines/fulltext/47/index.html)

3. SIGN 83. Prevention and Management of Dental Decay in the Pre-school Child. Scottish Intercollegiate Guidelines Network (2005) (www.sign.ac.uk/pdf/sign83.pdf)

4. Prevention and Management of Dental Caries in Children. Dental Clinical Guidance. Scottish Dental Clinical Effectiveness Programme (2010) (www.sdcep.org.uk)

5. Professionally applied topical fluoride: evidence-based clinical recommendations. American Dental Association Council on Scientific Affairs. Professionally applied topical fluoride: evidence-based clinical recommendations. J Am Dent Assoc 2006; 137: 1151-1159.

6. Petersson LG, Twetman S, Dahlgren $\mathrm{H}$, et al. Professional fluoride varnish treatment for caries control: a systematic review of clinical trials. Acta Odontol Scand 2004; 62: 170-176.

7. Azarpazhooh A, Main PA. Fluoride varnish in the prevention of dental caries in children and adolescents: a systematic review. / Can Dent Assoc 2008; 74: 73-79.

8. Carvalho DM, Salazar M, Oliveira BH, Coutinho ES. Fluoride varnishes and decrease in caries incidence in preschool children: a systematic review. Rev Bras Epidemiol 2010 13: 139-149.

9. Downs AM, Sansom JE. Colophony allergy: a review. Contact Dermatitis 1999; 41: 305-310.

10. Sharma PR. Allergic contact stomatitis from colophony. Dent Update 2006; 33: $440-442$.

Evidence-Based Dentistry (2013) 14, 72-73. doi:10.1038/sj.ebd.6400947 